\title{
A new atlas of the brain
}

\author{
When considering the location of human cognitive functions, \\ neuroscientists still refer to imprecise anatomical maps drawn up \\ almost a century ago. But not for much longer, says Alison Abbott.
}

A fter the death of Vladimir Ilyich Lenin in 1924, the Soviet authorities wanted to locate the seat of his 'genius'. So they sought the help of Oskar Vogt of the University of Berlin, a pioneering neuroanatomist and keen socialist. With the help of his wife Cécile and a few other close collaborators, Vogt had been the first to map the cerebral cortex according to its cellular anatomy.

Faced with Lenin's rather shrunken brain, Vogt was unable to identify any particular region that suggested extraordinary mental prowess. But he nonetheless declared Lenin a cognitive "athlete", blessed with a heightened ability to make connections in the brain due to the unusual density of a certain type of cell in the upper layers of his cortex.

Although neuroscientists no longer believe that broad concepts such as genius can be pinned down to precise brain locations, they do want to know where more clearly defined cognitive activities take place. Today, they approach this task armed with sophisticated machines for picturing the structure and activity of living brains. Yet they still relate the resulting images to an anatomy mapped out in 1909 by Vogt's Berlin collaborator Korbinian Brodmann ${ }^{1}$.

This is a serious limitation. Brodmann's map is at best imprecise and at worst downright wrong, says Karl Zilles of the C. \& O. Vogt Brain Research Institute at the University of Düsseldorf in Germany, whose team is now trying to produce a brain atlas for the twenty-first century. "Neuroscientists obviously need a more usable map," he argues.

Brodmann examined a handful of human brains, scrutinizing them under a light microscope to divide the cerebral cortex into around 50 numbered areas that he judged to be anatomically distinct. That was a formidable achievement for the time, but today the map is of limited use in interpreting the detailed functional and structural images produced by magnetic resonance imaging (MRI) and positron-emission tomography. "The ultimate goal is to ascribe structure to function and vice versa," says Ray Dolan, who heads the Functional Imaging Laboratory at University College London. Without a new map, this task remains largely a matter of guesswork.

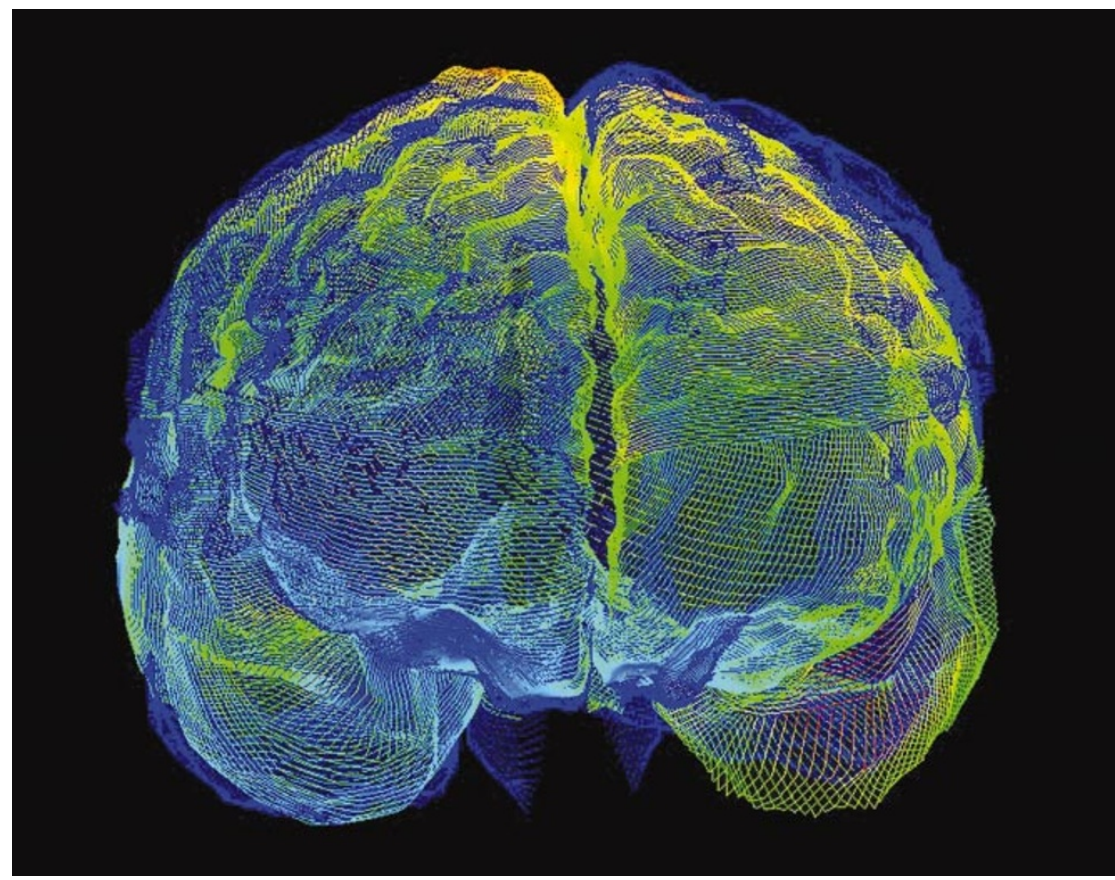

Spot the difference: Korbinian Brodmann's 1909 atlas (below left) does not account for variation between brains. In the composite image above, areas of high variation are shown in orange, yellow and green.

For one thing, Brodmann's twodimensional map represents only the surface of the cortex, whereas structural or functional brain imaging gathers data in three dimensions. What's more, the exposed surface of the cortex represents only one-third of the total surface - Brodmann did not map the other two-thirds, which are hidden in deep folds called sulci.

In an attempt to address some of the problems with Brodmann's map, most brain imagers today refer to an atlas published in 1988 by Paris-based neuroscientists Jean Talairach, of René Descartes University, and Pierre Tournoux, of the Val de Grace Hospi$\mathrm{tal}^{2}$. Talairach and Tournoux superimposed Brodmann's brain areas onto a three-dimensional description of one half, or hemisphere, of a single post-mortem brain. But this overlooks the fact that the brain is not symmetrical, and the map also overestimates the importance of sulci as landmarks of borders between distinct regions. "Worse still, it doesn't take into account the enormous variability in brain anatomy," says Zilles — neither
Brodmann nor Talairach and Tournoux realized that some brain areas vary in size by a factor of ten between different people.

\section{Time to think}

Over the past three decades, Zilles has developed a painstaking method for precisionmapping the cellular architecture of the human cortex. From the start, his goal has been to devise objective techniques that will not be biased by the neuroanatomist making the observations. "Brodmann's judgement about what constituted cortical areas was, by necessity, subjective - he could only look down his microscope and report," says Zilles, who is also head of the Institute of Medicine at the Jülich Research Centre, one of Germany's national laboratories.

Back in the 1970s, Zilles was viewed by many of his peers as an eccentric throwback to a bygone era. But today, the technology is in place to turn his vision into reality. The process begins at autopsy. For each brain being studied,Zilles's team makes an MRI scan before the organ is removed from the skull. They then embed the whole brain in a paraffin-wax block and cut it into slices just 20 micrometres thick - between 5,000 and 8,000 per brain. 
Each slice is stored on a numbered glass slide, and the researchers stain every fifteenth one to display cell bodies. Every sixtieth slice is then taken for computer measurement of cell densities. This gives an interval of 1.2 millimetres between each analysed slide, which is better than the resolution of current functional MRI. And should brain-imaging technology improve, says Zilles, it will be possible to return to the unexamined slides and boost the resolution of the map. Thus, as the brain's functions are pinned down more precisely, knowledge of brain architecture can expand in tandem.

By entrusting the analysis to computers, Zilles has removed human bias from the business of defining borders between brain regions. First, images of each slide are compared with the corresponding section of the original MRI scan to show how they have been distorted by their preparation. After the tissue is 'morphed' back to the size and shape that it had in situ, the images are then subdivided into $20-\mu \mathrm{m}$ grids, and the computer determines the proportion of stained cell bodies to unstained tissue in each square to calculate its 'grey-level index'. Finally, the computer maps borders between distinct anatomical areas by looking for sudden and statistically significant changes in these indices. "You can't rely on visual inspection to see where the boundaries are between different cortical areas," says Zilles. "You have to let the computer define the edges."

\section{Universal atlas}

The final product will involve 15 brains to generate a 'probabilistic map', against which any point selected from, say, an MRI image of a living brain can be said to lie within a particular structure with a certain probability. This tackles the issue of variation in brain anatomy between individuals.

Even with a dozen or so staff on the job, the atlas will take years to complete. But Zilles has already collaborated with brain imagers to identify several new cortical areas, which the researchers have defined both functionally and anatomically. These include two areas known as the ventral and anterior intraparietal areas (VIP and AIP), which had been identified in monkeys but were thought to have no human counterpart.

In monkeys, the VIP is associated with perception of motion independent of the type of sensory input — whether the moving object is seen, heard or felt. The monkey AIP, meanwhile, recognizes three-dimensional objects, also independently of the type of sensoryinput. In MRI studies of humans, the brain regions that lit up in response to each of these functions corresponded to distinct anatomical areas in Zilles' evolving brain atlas ${ }^{3,4}$.

Other neuroscientists are now collaborating with Zilles to try to pin down the anatomical locations of complex brain functions. For example, Per Roland of the Karolinska Institute in Stockholm, Sweden, has localized

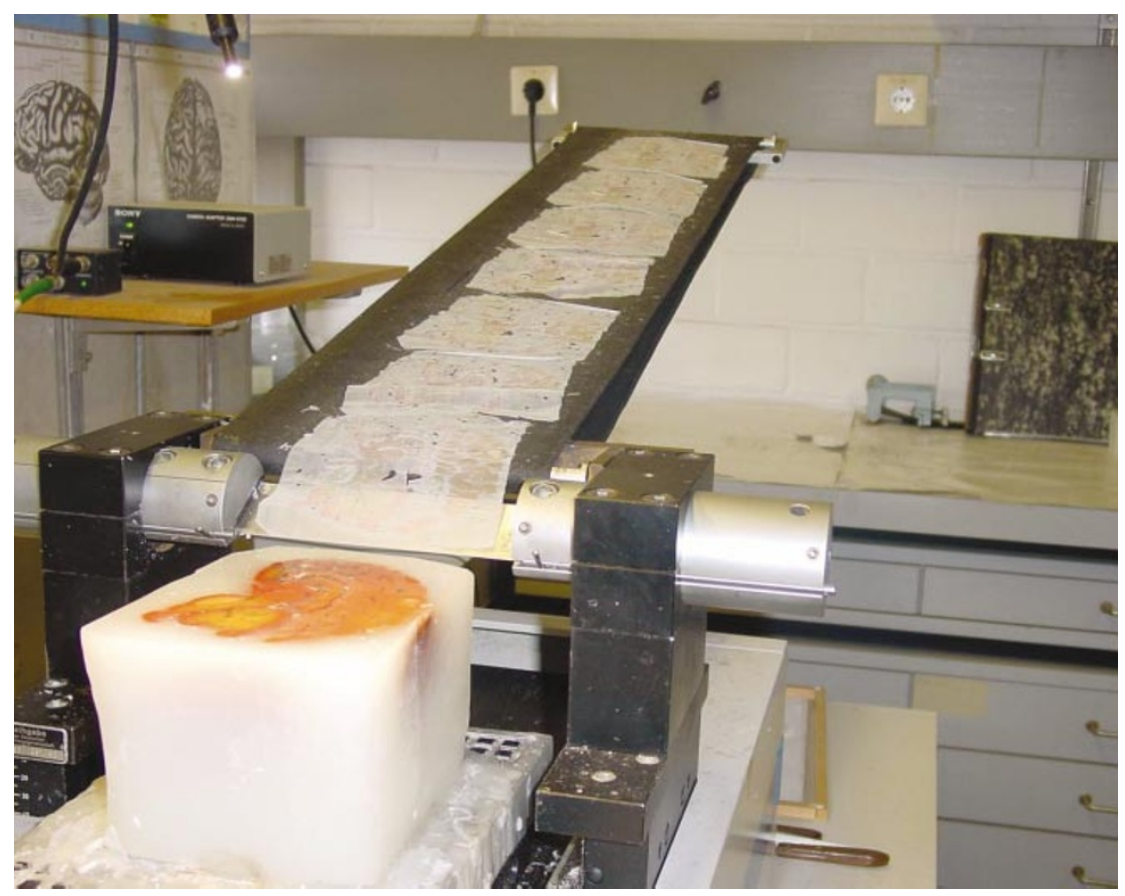

Head on the chopping block: Karl Zilles (below) carves wax-encased brains into ultrathin slices before using a computer to map their structure.

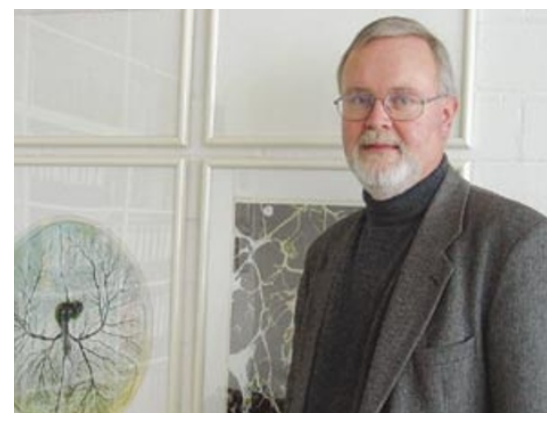

different components of the processing that takes place when we explore an object by touch, such as the detection of changes in curvature, to precise areas of the cerebral cortex ${ }^{5}$.

More recently, Zilles has begun to examine the distribution of receptors for different neurotransmitters, the chemical signals by which brain cells communicate with one another. Using chemicals that bind to specific receptors, he has found that distinct anatomical brain areas also have characteristic 'fingerprints' of receptor density ${ }^{6}$. This is particularly exciting, says Dolan, as a combination of cellular architecture and signalling chemistry may provide important clues as to how brain functions are regulated. "The functional-imaging field is also moving in this direction," he says.

Zilles is also a partner in the International Consortium for Brain Mapping (ICBM), based at the University of California, Los Angeles (UCLA), which is producing a database of thousands of structural images of brains from healthy volunteers as a resource for neuroscientists. 'Zilles' accurate, fine anatomy will provide essential information to users," says UCLA neurologist John Mazziotta, one of the ICBM's principal investigators.

Another ICBM group based at UCLA, headed by Arthur Toga and Jacopo Annese, is working to derive a similar map. They are staining sections of ten brains for myelin, the fatty substance that sheaths nerve fibres, rather than the cell bodies themselves. "It will complement the Zilles map," says Annese. "It will show the connections between all of the neurons, which is also very important for understanding function."

When such atlases are finished, brain imagers should be in a better position to interpret their data. "MRI is a poor camera for what is going on in the brain, but it's the best we can do with living brains," comments Alan Evans of the Montreal Neurological Institute at McGill University in Canada, who was one of the founding members of the ICBM. Together with detailed anatomical atlases, it should become a more powerful tool.

Such a resource would undoubtedly have benefited Vogt when studying Lenin's brain. But it would have had its drawbacks too Vogt might have found it rather more difficult to tell the Soviet authorities what they wanted to hear about their hero's cognitive abilities. Alison Abbott is Nature's senior European correspondent

1. Brodmann, K. Vergleichende Lokalisationslehre der Grosshirnrinde in ihren Prinzipien dargestellt auf Grund des Zellenbaues (J. A. Barth, Leipzig, 1909).

2. Talairach, J. \& Tournoux, P. Co-planar Stereotaxic Atlas of the Human Brain (Thieme Medical, New York, 1988).

3. Bremmer, F. et al. Neuron 29, 287-296 (2001).

4. Grefkes, C., Weiss, P. H., Zilles, K. \& Rink, G. R. Neuron 35, 173-184 (2002).

5. Bodegård, A., Geyer, S., Grefkes, C., Zilles, K. \& Roland, P. E. Neuron 31, 317-328 (2001).

6. Zilles, K. et al. Eur. Neuropsychopharmacol. 12, 587-599 (2002).

International Consortium for Brain Mapping

www.loni.ucla.edu/ICBM 